\title{
Pharmacotherapy for opioid dependence in jails and prisons: research review update and future directions
}

This article was published in the following Dove Press journal:

Substance Abuse and Rehabilitation

27 April 2016

Number of times this article has been viewed

\author{
Anjalee Sharma' \\ Kevin E O'Grady ${ }^{1,2}$ \\ Sharon M Kelly' \\ Jan Gryczynski' \\ Shannon Gwin Mitchell' \\ Robert P Schwartz'
}

'Friends Research Institute, Baltimore, ${ }^{2}$ Department of Psychology, University of Maryland, College Park, MD, USA
Correspondence: Robert P Schwartz Friends Research Institute, 1040 Park Avenue, Suite 103, Baltimore, MD 2I20I, USA

Tel + I 4I0 8373977

Fax + I 4107524218

Email rschwartz@friendsresearch.org
Purpose: The World Health Organization recommends the initiation of opioid agonists prior to release from incarceration to prevent relapse or overdose. Many countries in the world employ these strategies. This paper considers the evidence to support these recommendations and the factors that have slowed their adoption in the US.

Methods: We reviewed randomized controlled trials (RCTs) and longitudinal/observational studies that examine participant outcomes associated with the initiation or continuation of opioid agonists (methadone, buprenorphine) or antagonists (naltrexone) during incarceration. Papers were identified through a literature search of PubMed with an examination of their references and were included if they reported outcomes for methadone, buprenorphine, or naltrexone continued during incarceration or initiated prior to release in a correctional institution.

Results: Fourteen studies were identified, including eight RCTs and six observational studies. One RCT found that patients treated with methadone who were continued on versus tapered off methadone during brief incarceration were more likely to return to treatment upon release. A second RCT found that the group starting methadone treatment in prison versus a waiting list was less likely to report using heroin and sharing syringes during incarceration. A third RCT found no differences in postrelease heroin use or reincarceration between individuals initiating treatment with methadone versus those initiating treatment with buprenorphine during relatively brief incarcerations. Findings from four additional RCTs indicate that starting opioid agonist treatment during incarceration versus after release was associated with higher rates of entry into community treatment and reduced heroin use. Finally, one pilot RCT showed that providing extended-release naltrexone prior to discharge resulted in significantly lower rates of opioid relapse compared to no medication.

Conclusion: Reasons why uptake of these pharmacotherapies is limited in the US and relatively widespread in Europe are discussed. Recommendations for future research are outlined.

Keywords: methadone, buprenorphine, naltrexone, heroin, corrections, incarceration

\section{Introduction}

Incarceration is a relatively common experience among the estimated 15.6 million opioid-dependent adults in the world. ${ }^{1}$ In the US, it has been estimated that between $24 \%$ and $36 \%$ of opioid-dependent adults cycle in and out of jails each year. ${ }^{2,3}$ Incarceration of these individuals often results in opioid withdrawal syndrome, which, at a minimum, should be treated humanely. ${ }^{1,4}$ Beyond safe and effective opioid withdrawal treatment, there are three major opportunities to provide effective pharmacotherapy to inmates. First, inmates receiving opioid pharmacotherapy with either opioid agonists (eg, methadone or buprenorphine) or antagonists (naltrexone) in the community could 
be continued on their medications during brief incarceration. Second, inmates who experience withdrawal and who are either out of treatment at the time of incarceration or using contraband opioids during incarceration could be started and maintained on opioid pharmacotherapy. Finally, abstinent inmates with a long history of opioid dependence prior to incarceration and who are no longer physiologically dependent on opioids could be started on opioid agonist therapy (OAT) with either methadone or buprenorphine, or on the opioid antagonist extended-release naltrexone (XR-NTX) prior to discharge. Thus, incarceration presents a potentially important event for identifying and treating opioid-dependent adults.

Based on the principle that individuals should have access to the same treatment options during incarceration as those options available to them in the community, the World Health Organization (WHO) recommends that, at minimum, either methadone or buprenorphine should be made available to prisoners for maintenance treatment and opioid detoxification. ${ }^{1}$ The WHO also recommends that if countries can afford it, methadone, buprenorphine, and naltrexone should all be available to prisoners. While policies and practices regarding the use of these medications during incarceration vary from country to country, they are rarely used during incarceration in the US. ${ }^{5-7}$ In contrast, Canadian federal prisons can initiate methadone maintenance regardless of whether inmates were in community treatment prior to incarceration. ${ }^{8}$ Many nations in the European Union (EU) make methadone and/or buprenorphine available in jails and prisons. ${ }^{9,10}$ Methadone is also available to prisoners in Australia, the People's Republic of China, Iran, and elsewhere. ${ }^{11,12}$ However, in the US, Rikers Island Jail in New York City is one of the few jails that will initiate methadone treatment for individuals who were not receiving treatment in the community. This jail, along with others in Albuquerque (NM), New Haven (CT), and Baltimore (MD), is among the relatively few that will continue methadone treatment for detainees who were in communitybased treatment at the time of arrest. A growing number of US jails and prisons are making XR-NTX available. ${ }^{13}$ This longacting injectable medication, which provides a blockade against the effects of opioids for $\sim 1$ month, can be administered just prior to release for individuals who are abstinent from opioids for a period of approximately 1 week.

Initiation of opioid pharmacotherapy during incarceration could play an important role in safeguarding both individual and public health, and safety, given the high financial cost of opioid dependence, ${ }^{14}$ high post-release relapse rates, ${ }^{15}$ drug injection, human immunovirus (HIV) risk, ${ }^{16}$ and overdose death of newly released inmates. ${ }^{17}$ Despite the fact that in the late $1960 \mathrm{~s}$, the US was the first country to adopt widescale use of methadone treatment in the community and to have a random assignment trial of methadone treatment in a jail, ${ }^{18}$ it lags behind many countries in the developed world in providing adequate pharmacotherapeutic treatment of opioid use disorder and in implementing opioid pharmacotherapies during incarceration. ${ }^{19}$ The purpose of this paper is to provide a review of the extant peer-reviewed publications on the use of pharmacotherapy for opioid dependence in correctional institutions in order to update a prior such review of opioid agonist treatments, ${ }^{20}$ to discuss reasons that might explain why the US has not widely adopted this approach, and to propose future research directions.

\section{Methods}

Inclusion criteria for this review were English language peer-reviewed publications of randomized controlled trials (RCTs) or observational studies (included due to the small number of RCTs) with results of initiating or continuing an opioid agonist or antagonist treatment during incarceration for adults ages 18 years and older.

An electronic search was conducted in PubMed using the search terms "methadone initiation in jail," "methadone initiation in prison," "buprenorphine initiation in jail," "buprenorphine initiation in prison," "naltrexone initiation in jail," "naltrexone initiation in prison," "methadone jail," "buprenorphine jail," "naltrexone jail," "methadone prison," "buprenorphine prison," and "naltrexone prison" yielding 287 publications. Papers were only included if they were published in English and if they assessed the initiation or continuation of opioid agonist or antagonist pharmacotherapy in a correctional setting. The final literature search was conducted in the summer of 2015, of which the findings are reported herein. The lead author read the title and abstract to ascertain whether the study fits the focus of this paper. Papers that clearly did not pertain to the subject matter or that did not report the results of original research (eg, literature review, thought pieces, or papers that dealt exclusively with outpatient data) were immediately excluded, although bibliographies of such papers were examined in order to ensure that all relevant studies were found. The remaining papers were obtained and thoroughly read in order to determine if they met criteria for inclusion in this review. A total of ten RCTs were found through this literature search. One RCT found in the literature search was excluded from this report because it examined naltrexone implants, ${ }^{21}$ which are only approved for use in Russia where opioid agonist medications are not permitted in the community, and hence, its findings 
would not be of widespread use at this time. A second RCT that was conducted by our group with levo-alpha acetylmethadol was excluded because this medication is no longer available. $^{22}$ Thus, a total of eight RCTs were included in this review. In addition, there were six longitudinal studies that were identified.

Studies were categorized according to whether medications were 1) continued from treatment in the community, 2) initiated during opioid withdrawal for opioid agonist maintenance treatment, or 3) initiated after withdrawal was completed for the prevention of relapse. Within these three categories, reports were further divided between RCTs and observational reports and listed chronologically. Studies were summarized by listing their 1) setting, 2) sample size, 3) study arms, 4) participants' sex, 5) average age of participants, 6) participants' race/ethnicity, and 7) follow-up time frame. As available, outcomes for each of these studies were categorized based on 1) percentage of participants who entered assigned treatment post release, 2) reincarcerations, 3) self-reported opioid misuse post release, 4) injection drug use post release, 5) retention in treatment, and 6) study limitations.

\section{Results}

Table 1 summarizes the demographics of each study's participants included in this review. Table 2 summarizes the primary outcomes of these studies.

\section{Continuing methadone maintenance treatment for patients enrolled in such treatment in the community at the time of arrest}

Rich et $\mathrm{al}^{23}$ completed an RCT in Rhode Island, USA, between 2011 and 2013 that compared methadone continuation for individuals enrolled in methadone maintenance treatment (MMT) at the time of arrest versus a forced, relatively slow, methadone dose taper during incarceration. There were a total of 223 participants randomized to either methadone continuation $(n=114)$ or forced methadone dose taper $(n=109)$. Males comprised $78 \%$ of the sample, $81 \%$ of participants were Caucasian, and the mean age was 34 years (standard deviation $[S D]=8.4$ ). Participant data were gathered at 1-month post release with an $88 \%$ follow-up interview rate. At follow-up, 106 (96\%) of the methadone continuation group had reentered treatment, compared to 68 (78\%) of the forced dose taper group $(P=0.001)$. Twelve participants $(11 \%)$ from the methadone continuation group and eight $(9 \%)$ from the forced dose taper group were reincarcerated.
The proportion of participants reporting opioid use at the 1-month follow-up was more than twice as high in the forced taper group compared to the continuation group (16 [18\%] vs 9 [8\%], respectively; $P=0.033)$. Similarly, 19 (17\%) individuals in the methadone continuation group reported intravenous drug use at follow-up, compared to $28(32 \%)$ in the forced taper group $(P=0.016)$. Neither retention in treatment nor urine drug screening results were reported. While the results demonstrated the superiority of continuing methadone during incarceration, findings would likely have been even stronger had all of the members of the control group been completely withdrawn from medication by the time they were released, as is commonly the case in the US.

\section{Initiation of opioid agonist treatment for inmates out of treatment}

Five papers describing studies of initiating opioid agonist treatment with either methadone or buprenorphine were reviewed: two RCTs (Table 1) and three longitudinal studies.

\section{Randomized clinical trials}

In an Australian prison in New South Wales, Dolan et al conducted an RCT from August 1997 to October 1998 to determine whether methadone maintenance reduced heroin use, syringe sharing, and/or incidence of HIV or hepatitis C during incarceration. ${ }^{24} \mathrm{~A}$ total of 382 were randomized to either MMT $(n=191)$ or a no-treatment control condition $(n=191)$. The entire sample was male, and race/ethnic data were not reported. The average age of participants was 27 years $(\mathrm{SD}=6)$. Follow-up interviews were conducted in prison, 4 months following baseline, with 253 (66.2\%) participants (because some participants were released prior to follow-up). Compared to the control group, participants in the MMT group were significantly less likely to report heroin injection ( $32 \%$ vs $74 \% ; P<0.001)$ and syringe sharing (20\% vs 54\%; $P<0.001)$. However, there were no significant differences in opioid-positive hair test results (39 [31\%] vs 43 [37\%] in the MMT and control groups, respectively). This study's limitations included an all-male sample and no reported postrelease data by study condition. However, a subsequent 4-year follow-up report indicated that participants who were in methadone treatment in the community had a lower mortality rate than participants who were not enrolled in treatment. ${ }^{25}$

Magura et $\mathrm{al}^{26}$ published an RCT comparing the initiation of methadone and buprenorphine treatment among jail inmates to compare their outcomes after release. Participants 


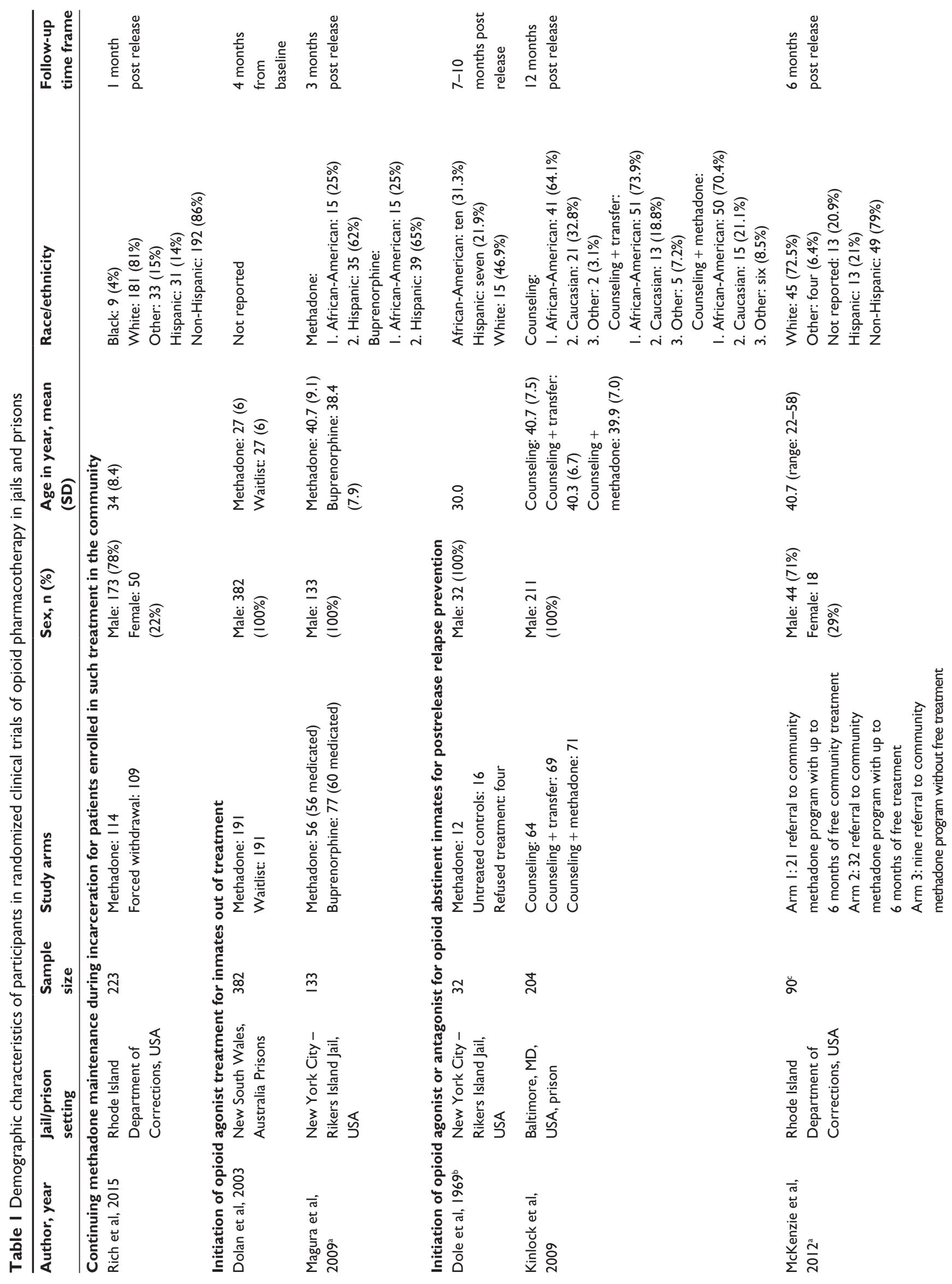




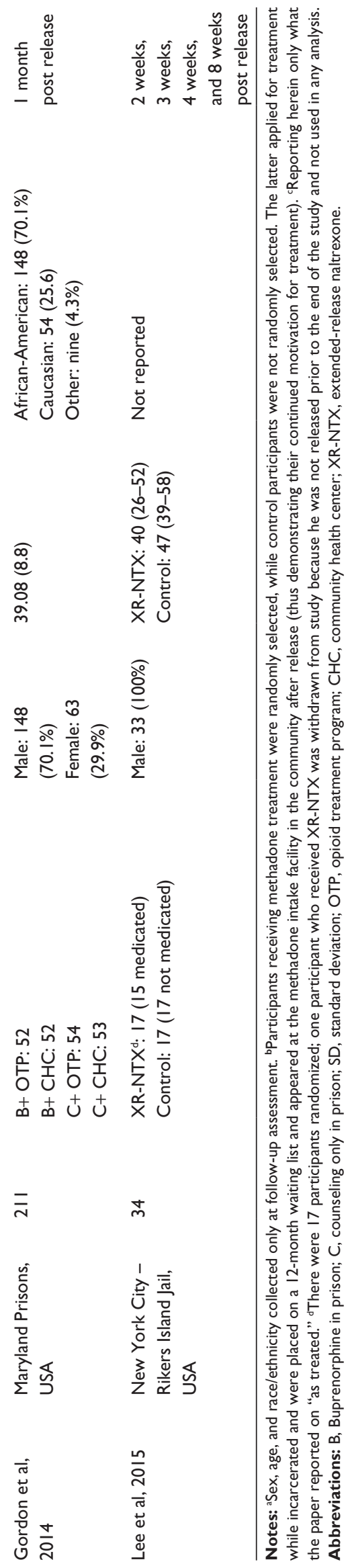

were enrolled between August 2006 and 2007 at the Rikers Island Jail's methadone program, which was started in the mid-1980s in response to the HIV epidemic. ${ }^{27,28}$ Opioiddependent males who were not in treatment at the time of arrest were assigned using treatment-adaptive randomization to either buprenorphine or methadone treatment. A total of 56 men were randomly assigned to and received methadone and 77 men were assigned to buprenorphine, of whom 60 received their assigned medication. As shown in Table 1, the average age of the methadone and buprenorphine groups was 40.7 years $(\mathrm{SD}=9.1)$ and 38.4 years $(\mathrm{SD}=7.9)$, respectively. Seven participants were terminated from treatment in jail for attempted medication diversion (six [10\%] in the buprenorphine group and one [1.8\%] in the methadone group). At 3 months post release (Table 2), the buprenorphine group was significantly more likely than the methadone group to have entered their assigned postrelease treatment ( $48 \%$ vs $14 \% ; P<0.001)$. The buprenorphine and methadone groups did not differ significantly in terms of reincarceration rates ( $40 \%$ vs $50 \%$, respectively) or any self-reported heroin use (53\% vs 66\%, respectively) at the 3-month follow-up. There were a number of limitations to this study, including no report of community treatment retention or urine drug screening. The sample was exclusively males, and there were acceptable follow-up rates $(70 \%)$.

\section{Longitudinal studies}

Between November 1988 and April 1990 at Rikers Island Jail in New York City, Magura et $\mathrm{al}^{28}$ conducted the first longitudinal study of out-of-treatment jail inmates who were initiated on MMT. The study compared outcomes at a median of 6.5 months post release of a systematically selected cohort of 446 male and female patients starting methadone maintenance $(n=308)$ at Rikers Island to those of a second cohort that received methadone detoxification $(\mathrm{n}=138)$. Follow-up assessments were conducted with only 250 (56.1\%) of the 446 patients. The MMT group entered and were retained in methadone treatment at higher rates (85\%) than the control group (37\%) and reported higher rates of heroin abstinence post release (31\% vs $22 \%$, respectively). Study limitations included lack of random assignment, no urine drug screening, and low follow-up rates.

In France, buprenorphine can be prescribed by all physicians (including those working in prisons) as of 1996, and methadone has been available in prisons since 2002 . A longitudinal study was conducted by Marzo et $\mathrm{al}^{29}$ in 2003 and 2004 in 47 French remand prisons (where newly detained individuals are housed). This study examined 


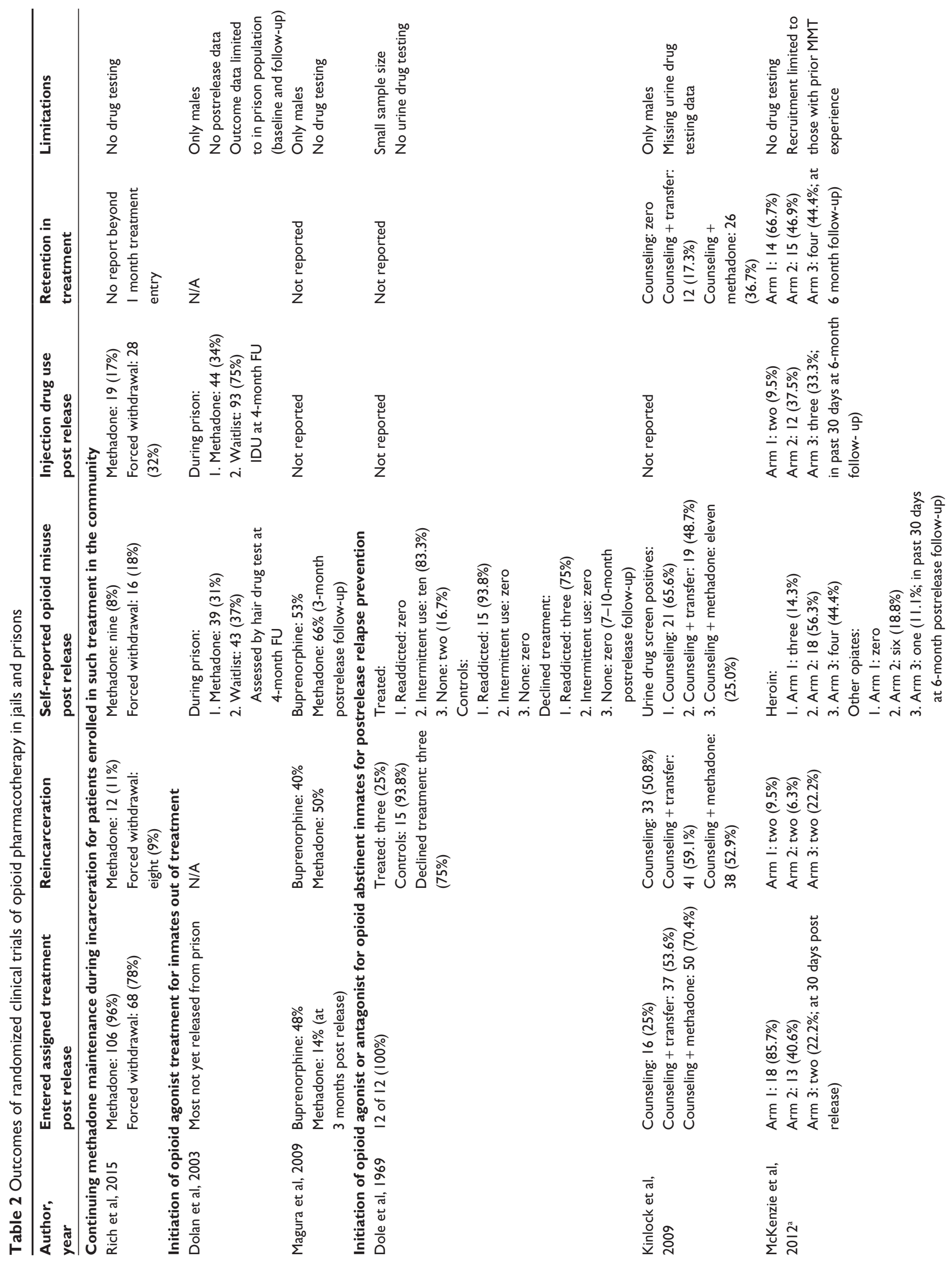




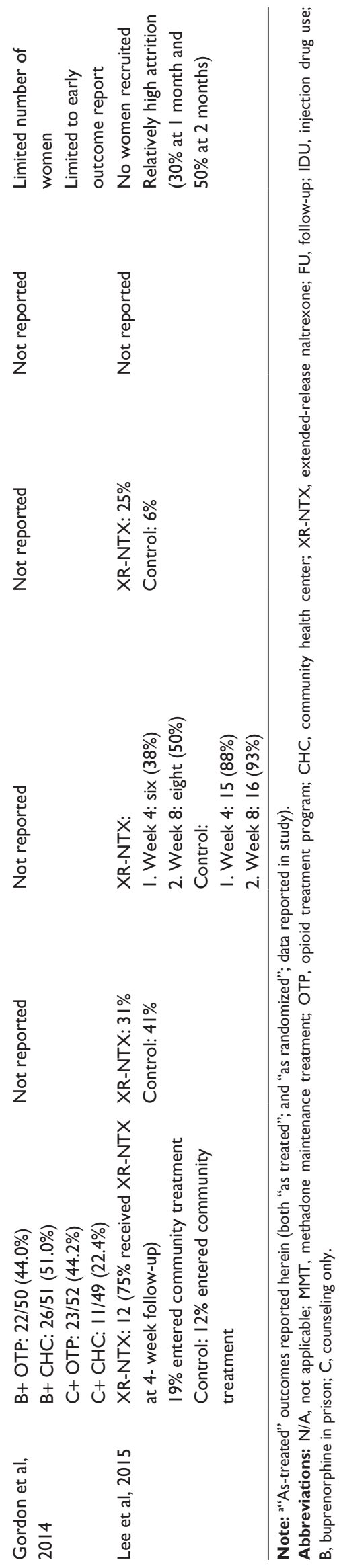

3-year reincarceration rates of 507 male and female opioid-dependent inmates. Baseline data were obtained at admission, while 3-year follow-up reincarceration data were obtained through database searches. The majority of the sample was males $(96.3 \%)$, the mean age of participants was 30.8 years $(\mathrm{SD}=6.4), 78 \%$ had a prior incarceration, $54.1 \%$ had injected heroin in their lifetime, and $69.3 \%$ reported being on opioid agonist treatment in the month prior to incarceration. Opioid agonist pharmacotherapy was provided to 394 (77.7\%) study participants. The reason(s) that the remainder of the sample did not receive medication was(were) not described. Maintenance treatment was started for 69 of 394 (17.5\%) out-of-treatment individuals at the time of incarceration and continued for 324 of 394 $(82.2 \%)$ individuals who had been receiving such treatment at the time of incarceration. The majority of participants (73.6\%) were treated with buprenorphine, and the remainder were treated with methadone. Of the 478 individuals who were subsequently released from prison, $238(49.8 \%)$ were reincarcerated at least once during the 3-year follow-up period. Multivariate analysis found that a history of prior incarceration and benzodiazepine use within the month prior to the index incarceration, but not maintenance therapy, was associated with reincarceration. This study had a number of limitations, including the lack of participant interviews, urine drug screening, and postrelease treatment entry and retention data at follow-up.

Switzerland's largest remand prison (located in Geneva) has been offering opioid agonist treatment for $\sim 20$ years. Favrod-Coune et $\mathrm{al}^{30}$ retrospectively reported on the dosing practices and patient characteristics of this program. The mean age of the entire sample of current opioid users who were detained and awaiting trial between January 2007 and December $2007(\mathrm{n}=241)$ was 29.6 years ( $\mathrm{SD}=7.1)$. Ethnicity was $28.9 \%$ Western European, 27.5\% North African and Middle Eastern, 20.1\% Sub-Saharan African, and 23.5\% others. For this study, 233 inmates who used opioids and had complete data were identified, of whom $95.3 \%$ were males and 39.9\% injected drugs. Of these individuals, 166 (71.2\%) were considered opioid dependent and treated with either methadone or buprenorphine. Approximately $20 \%$ had been receiving opioid agonist treatment in the community prior to incarceration. The only outcome data reported on the 166 people who received OAT during incarceration were that $49.7 \%$ received follow-up care in the community after their release. Of the 67 individuals who did not receive OAT during incarceration, only 19 (28.4\%) received follow-up care in the community. This study had several limitations including 
lack of participant interviews and urine testing, and no data on community treatment retention.

\section{Initiation of opioid agonist or antagonist for opioid abstinent inmates for postrelease relapse prevention}

We identified seven publications regarding the initiation of opioid agonists or antagonists prior to release, including five RCTs and two longitudinal studies.

\section{Randomized trials of opioid agonists}

In a pioneering study, Dole et $\mathrm{al}^{18}$ sought to determine inmates' level of interest in, and effectiveness of, initiating methadone maintenance prior to release from a New York City jail (Rikers Island). The inclusion criteria were having at least 5 years of heroin addiction, five or more prior convictions, and a scheduled release from jail between January 1, 1968, and April 30, 1968. Individuals were excluded if they were committed to the custody of the Addictions Service Agency. Of the 165 male inmates screened for participation, $116(70 \%)$ applied for treatment. As shown in Table 1, the study sample consisted of 32 men. Initially, 16 were randomly selected to begin methadone treatment $\sim 10$ days prior to release; however, four from this group declined treatment. Another 16 who had an expressed interest in treatment (untreated controls) were placed on a waiting list for the 12-month duration of the study. Participants had a mean age of 30 years (SD $=5.08$ ) and were $31.3 \%$ African-American, 21.9\% Puerto Rican, and $46.9 \%$ of European descent. Because the participants were not physiologically dependent on opioids at the time of dose induction, methadone was started at only $10 \mathrm{mg}$ and was increased slowly to $\sim 35 \mathrm{mg}$ by the time of release. No other services were provided during methadone treatment in jail. Outcome data obtained from methadone program records and follow-up interviews conducted at 3-month intervals post release were examined between 7 months and 10 months post release to determine selfreported opioid use and whether they were rearrested at follow-up. Despite ten of $12(83.3 \%)$ treated participants reporting having used heroin intermittently post release (Table 2), none of these 12 men reported readdiction (eg, regular daily use) to opioids. Three men in this group were reincarcerated. In contrast, 15 of 16 control participants became readdicted and reincarcerated, and the other was lost to follow-up. Three of the four individuals who refused treatment became readdicted to opioids and were reincarcerated, and one was lost to follow-up. This study demonstrated that inmates had an interest in initiating methadone prior to release. It also demonstrated the potential utility of initiating methadone treatment prior to release in a population no longer physiologically dependent at the time of treatment initiation. The study had a number of limitations, including a small sample size and no urine drug screening.

A second RCT examining the initiation of MMT prior to release from prisoners recruited participants from September 2003 to June 2005 from a prison in Baltimore, MD, USA. Participants were 211 males with a history of heroin dependence who were not physiologically dependent at the time of study enrollment. ${ }^{15}$ Participants were randomly assigned on a 1:1:1 basis to counseling only with a passive referral to treatment in the community/counseling with referral to guaranteed MMT entry in the community/ counseling with MMT in prison, with guaranteed MMT continuation in the community. Because participants were not opioid dependent at study entry, methadone treatment was started at a low dose $(5 \mathrm{mg} / \mathrm{d})$ that increased quite slowly at a rate of $5 \mathrm{mg} /$ week to a target of $60 \mathrm{mg}$ methadone daily. Follow-up interviews were conducted at 1 month, 3 months, 6 months, and 12 months post release. By 1 month post release, there was a significant difference in community methadone treatment entry such that only $7.8 \%$ had entered treatment in the counseling only condition, compared to $50 \%$ in the counseling with postrelease referral to MMT in the community, and $68.6 \%$ in the counseling with methadone in prison condition (all $P_{S}<0.05$ ). ${ }^{31}$ Outcome data were obtained on 204 (96.7\%) participants, although it was not possible to obtain $89(43.6 \%)$ urine samples at follow-up. All participants were males with a mean age of 40.3 years ( $\mathrm{SD}=7.1$ ), and $69.6 \%$ were African-American and $24.0 \%$ were Caucasian. There was a significant difference between conditions of days in treatment during the year post release. The counseling only group had 23.1 days in treatment ( $\mathrm{SD}=72.5$ ), counseling with postrelease referral to MMT in the community had 91.3 days in treatment $(\mathrm{SD}=144.6)$, and counseling with methadone in prison had 166.0 days in treatment $(\mathrm{SD}=166.4$; in all pairwise comparisons, $P<0.01)$. Just over half of all participants were reincarcerated during the 1-year follow-up, with no significant differences between conditions. Both the counseling only (65.6\%) and counseling in prison with postrelease referral to MMT in the community (48.7\%) conditions had significantly higher rates of opioidpositive urine tests at the 12-month follow-up compared to the counseling with methadone in prison condition $(25 \%$; $P=0.001$ and 0.008 , respectively). This study had a number of limitations, including missing data for urine drug screening 
(43.6\% of the samples were missing for a variety of reasons) and a male-only sample.

A third RCT was conducted by McKenzie et $\mathrm{al}^{32}$ to further examine the effectiveness of MMT prior to release among sentenced inmates with a history of opioid dependence who were not physiologically dependent on opioids. This study recruited participants between October 2006 and February 2009 from the Rhode Island Department of Corrections, and randomly assigned 90 participants to 1) methadone initiation prior to release with short-term subsidized MMT in the community (12 weeks for free and 12 weeks for half price), 2) referral to MMT in the community with the same subsidy as group 1, or 3) referral to community MMT without subsidy. Inclusion criteria were as follows: 1) inmates 18 years or older who had sentences of $\leq 2$ years, 2) at least one prior MMT experience, 3) injection heroin use prior to incarceration or being on MMT prior to incarceration, 4) scheduled release date at least 28 days after study enrollment, 5) at least one prior drug-related incarceration, and 6) willingness to provide two verifiable contacts. Participants assigned to methadone initiation were not physiologically dependent at enrollment, and therefore, were started on a low dose of methadone ( $5 \mathrm{mg} /$ day) that increased by $2 \mathrm{mg} / \mathrm{d}$ until reaching their individualized target dose or their release from jail. The average dose of methadone at release was $33 \mathrm{mg}$ (range: $5-38 \mathrm{mg} / \mathrm{d}$ ). As shown in Table 1, participants (reported for only those 62 [70\%] who had completed a 6-month follow-up) had a mean age of 40.7 years (range: $22-58$ ) and were $71 \%$ males, $72.5 \%$ whites, $6.4 \%$ others, and $21 \%$ Hispanics, and for $20.9 \%$, race was not reported. During the course of the study, a grant to provide free treatment for all participants was obtained by Rhode Island, and the third study arm (treatment without subsidy) discontinued enrollment. Furthermore, some participants assigned to group 1 were unable to start MMT prior to release and were offered methadone after release (thus, shifting to group 2). Because of these group "cross-overs," the investigators, in addition to an intent-to-treat analysis, conducted an "as treated analysis" as shown in Table 2 to better reflect the outcomes of the actual services received rather than assigned. The group that initiated methadone prior to release compared to the other groups was significantly more likely to enter methadone treatment within 30 days of release $(P<0.001)$, although 6 -month treatment retention was not significantly different between the groups (Table 2). The group that initiated methadone in jail compared to the other groups reported lower rates of heroin use in the 30 days prior to the 6-month postrelease assessment $(P=0.008)$. Two participants in the group assigned to begin methadone after release died of opioid overdose shortly after release. There are a number of limitations in this study, including recruitment limited to those individuals who had prior MMT experience, lack of biological testing for drug use, and cross-over from assigned study conditions.

A fourth RCT was conducted in a Baltimore, MD, USA, prison to examine buprenorphine initiation prior to release among opioid-dependent adult prisoners who were no longer physiologically dependent on opioids. It assessed the initiation of buprenorphine in prison versus in the community and also examined whether outcomes differed between participants randomly assigned to postrelease treatment at an opioid treatment program (OTP) or a community health center (CHC). ${ }^{33} \mathrm{~A}$ total of 213 participants were recruited between September 2008 and July 2012 and randomly assigned to one of four groups: 1) buprenorphine treatment initiated in prison and continued at an OTP $(n=53), 2)$ buprenorphine treatment initiated in prison and continued at a $\mathrm{CHC}(\mathrm{n}=53), 3)$ counseling only in prison and initiation of buprenorphine at an OTP ( $\mathrm{n}=54)$, and 4) counseling only in prison and initiation of buprenorphine at a $\mathrm{CHC}(\mathrm{n}=53)$. Two participants were excluded from the study just after randomization, and therefore, data from 211 participants were used in the analysis. Participants' mean age was 39.08 years $(\mathrm{SD}=8.8)$, and most were men (70.1\%) and African-American (70.1\%). Initial outcome data were obtained on $96.7 \%$ of participants within 10 days post release. Notwithstanding the fact that 19 participants $(17.5 \%)$ assigned to receive buprenorphine in prison were tapered off medication because of attempted medication diversion, the groups assigned to start buprenorphine in prison were more likely to enter community treatment compared to the groups assigned to start buprenorphine post release $(47.5 \%$ vs $33.7 ; P=0.01)$. There were no significant differences in treatment entry by postrelease treatment site. This paper did not report community outcomes beyond the 10-day treatment entry data.

\section{Longitudinal studies of opioid agonist treatment}

The feasibility of providing buprenorphine treatment in a minimum security prison in Puerto Rico was studied among 45 male opioid-dependent prisoners. ${ }^{34}$ The 1-month follow-up rate was $93.3 \%$. The majority of participants entered community treatment at a physician's office post release $(n=35$; $77.8 \%$ ), and 33 of these individuals continued buprenorphine at 1-month post release. Participants who remained in treatment at 1-month post release compared to participants who were out of treatment had greater reductions from prerelease baseline to follow-up interview in the median number of days 
of self-reported use of heroin $(P=0.01)$ and cocaine $(P=0.04)$, and lower rates of opioid-positive urine test results $(25 \%$ vs $33.3 \%$, respectively). There were no reincarcerations at the 1-month follow-up.

A study conducted in the Rhode Island Department of Corrections (USA) by Zaller et $\mathrm{al}^{35}$ examined the feasibility of initiating buprenorphine prior to release from incarceration. The study design was initially a longitudinal pilot study with a 6-month follow-up, but due to logistical issues, two groups emerged $(\mathrm{N}=44)$ : one that was medicated prior to release $(n=12)$ and the other that was medicated only after release $(\mathrm{n}=32)$. At the 6-month follow-up, 36 participants $(82 \%)$ were assessed. In all, eleven of 12 (92\%) participants who started buprenorphine prior to release had entered community treatment compared to 25 of $32(78 \%)$ who were unable to initiate medication prior to release $(P=0.30)$. The mean number of weeks in community treatment was 20.3 weeks for those initiated on buprenorphine while incarcerated compared to 13.2 weeks for those participants who were initiated post release $(P=0.007)$. Overall, initiation of buprenorphine while incarcerated was shown to be feasible and to be associated with longer community treatment retention compared to starting medication after release.

\section{Studies of using opioid antagonists for relapse prevention \\ Randomized trial of opioid antagonist medication}

In the first US report of the use of the opioid antagonist XR-NTX to prevent relapse among jail inmates, Lee et $\mathrm{al}^{36}$ conducted a two-arm RCT in which 34 sentenced adult male prisoners in the Rikers Island jail with a known release date were recruited between January 2010 and April 2013 and were randomly assigned to receive either an intramuscular injection of XR-NTX prior to release or a treatment-as-usual control group without medication. Inmates were eligible for the study if they were 18 years or older, met DSM-IV criteria for opioid dependence prior to incarceration, had a known release date, were neither receiving nor intending to receive methadone or buprenorphine treatment, had not recently used an opioid, had a negative urine opioid test, did not have opioid withdrawal symptoms in response to a naloxone (Narcan) challenge, were not pregnant, and had liver function tests no more than three times the normal level. Eligible participants who provided informed consent received a research assessment and some brief drug abuse counseling and aftercare referral information and were then randomly assigned to receive XR-NTX prior to release with the possibility of having two additional injections at 30 days and 60 days after release or to a control group. In the XRNTX group, two of 17 participants refused medication, one of 17 was medicated but withdrawn from the study because his release was delayed, and 15 of 17 received an injection of XR-NTX during incarceration. None of the 17 participants assigned to the control group received XR-NTX. The average age of the participants was 40 years in the XR-NTX group and 47 years in the control group $(P<0.01)$. In the XR-NTX group, 12 of the 16 participants who were released $(75 \%)$ received a second naltrexone injection at 1-month post release. As shown in Table 2, at the 4-week follow-up, there was a significant difference in the primary outcome of opioid relapse (defined as $\geq 10$ days of opioid use and opioidpositive urine drug testing). Six participants (38\%) of the XR-NTX group and 15 (88\%) of the control group relapsed $(P<0.004)$. There were no significant differences between conditions in the secondary outcomes of postrelease injection drug use, cocaine use, participation in community drug treatment, or reincarceration. The study had a number of limitations, including relatively small sample size with a high attrition rate (30\% at 4 weeks and $50 \%$ at 8 weeks).

\section{Longitudinal study of opioid antagonist medication}

In an observational pilot study by Gordon et al, ${ }^{37} 27$ adult male and female prerelease prisoners were given an injection of XR-NTX prior to release and were offered up to six monthly injections in the community. More than one-third of participants (37\%) completed all six injections. Participants who completed the full course of treatment were less likely to submit a positive urine opioid screen at follow-up in the community ( $0 \%$ vs $62.5 \%$, respectively; $P=0.003$ ). This pilot study demonstrated the feasibility of beginning XR-NTX in prison and continuing it upon release.

\section{Discussion}

Since Dole et $\mathrm{al}^{18}$ study was published $\sim 50$ years ago, which examined initiation of methadone prior to release from jail to prevent relapse, relatively few prospective clinical studies of opioid pharmacotherapy in correctional settings have been published. Although expanding, research data on the evidence for the effectiveness of pharmacotherapy for opioid dependence in correctional settings remain limited. The literature review by Hedrich et $\mathrm{al}^{20}$ (which also included nonpeerreviewed government reports of evaluations) found that methadone treatment in prison reduces heroin use and needle sharing and is associated with increased treatment entry post release. However, this review noted that the evidence for other favorable outcomes is either weaker (reducing heroin use 
post release), equivocal (reducing crime and incarceration), or insufficient (reducing HIV infection or mortality). Since this publication, several new RCTs have been published. First, a large RCT demonstrated a higher rate of return to treatment following incarceration for participants who were enrolled in methadone treatment at the time of arrest and assigned to continuing methadone treatment compared to participants who received a dose taper during detention. ${ }^{23}$ A second RCT found that starting buprenorphine in prison increases the likelihood of attending treatment post release compared to similar guaranteed treatment entry post release for participants who were assigned to receive counseling without medication in prison. ${ }^{33}$ There have also been two studies with XR-NTX, a pilot RCT that showed that providing this medication prior to discharge resulted in significantly lower rates of opioid relapse compared to no medication ${ }^{36}$ and an observational pilot study that showed the feasibility of initiating XR-NTX prior to release. ${ }^{37}$

Most of the extant peer-reviewed literature examined relapse prevention strategies initiating medications for individuals who were opioid abstinent and no longer tolerant in order to reduce the risk of relapse and overdose death post release. ${ }^{17}$ Few US jails and prisons initiate pharmacotherapy for prisoners despite the recommendations of the WHO. ${ }^{1}$ This is unfortunate because postrelease relapse rates following extended prison sentences are high, ${ }^{31}$ and the trials of using medications for relapse prevention indicate that there appear to be significant benefits to starting opioid agonist or antagonist medications prior to release in terms of increased entry into community treatment and reduced heroin use at follow-up. However, more research is needed comparing different approaches to increasing treatment entry post release.

As noted in the introduction, notwithstanding the fact that the US was the first country to make widespread use of methadone maintenance therapy in the community and to study its use during incarceration, it lags behind many countries in providing medications during incarceration to treat opioid dependence. The reasons for this are complex and may be influenced by a number of intertwined issues. First, in the UK, EU, and elsewhere, it is widely recognized that prisoners have the right to the same health care in prison as in the community. ${ }^{10,38}$ This principle is consistent with the position of the WHO that either methadone or buprenorphine should be available to prisoners for maintenance and management of opioid withdrawal, and that best practice recommendations are to ensure the availability of methadone, buprenorphine, and naltrexone for prisoners. ${ }^{1}$ Thus, many countries have implemented the full range of methadone and buprenorphine treatment in correctional institutions to match their community treatment spectrum.

The evidence from clinical trials to support the WHO recommendation varies depending on the purpose of the use of the medication (initiating at arrest, during incarceration or just prior to release, continuing community treatment at the time of arrest) and the type of medication. Thus, to date three important questions have been examined in just one trial each. These questions are 1) is it better to continue methadone or taper off methadone during brief incarcerations for people who are taking methadone at the time of incarceration, 2) which medication during incarceration (methadone vs buprenorphine) affords superior community outcomes after release, and 3) does methadone maintenance in prison afford superior in-prison outcomes as compared to no medication? The evidence supporting naltrexone use stems only from one small RCT with limited community follow-up data. Finally, evidence for starting methadone or buprenorphine prior to versus after release from longer prison sentences has more robust support from four trials. One could easily (and rightly) argue that inmates should have access to approved medications that are available in the community. However, given the unique circumstances of incarceration and challenges linking patients to community services after release, community research findings may not automatically generalize to correctional settings. At the very least, it is clear that important implementation questions regarding the delivery of these services within the correctional institutions and upon discharge require clarification and further study. It is also true that in the absence of strong direct evidence in favor of using these medications during incarceration, it may be difficult to convince correctional administrators to adopt these treatments in light of the cost and logistical challenges.

In some countries such as the US, providing health care to prisoners may not typically be entirely determined by community standards. ${ }^{39}$ Whether methadone treatment (or presumably other newer pharmacotherapies for opioid dependence) is required to be provided during incarceration has been the subject of numerous lawsuits and may be protected under various federal laws. ${ }^{40,41}$ However, to date the issue has not been settled. Further complicating the US situation is the decentralized nature of its criminal justice system, much of which is under the jurisdiction of a patchwork of states and municipalities. US jails detain individuals prior to trial as well adjudicated individuals with sentences of, generally, $<1$ year, while prisons house adjudicated individuals whose sentences generally are $>1$ year. Outside the US, facilities housing detainees are called remand prisons in some countries. Jails 
in the US, unlike prisons (with few exceptions), are operated by the local cities, counties, or Indian tribal authorities and report to political leadership at the local level, which is quite independent of the state. Each of the 50 US states has its own separate prison system operated by a department of corrections. Some prisons are even run by for profit companies on behalf of states. In addition, there is a separate federal bureau of prisons that operates federal penal institutions across the country. Hence, there is no one corrections entity that could direct all of these institutions to provide pharmacotherapy.

In contrast to the US, most developed countries have universal health care in the community, and drug abuse treatment is a component of their health care system. The US does not have a universal health care system, but rather a medley of public and private arrangements that vary widely from state to state. Therefore, drug abuse treatment coverage is by no means universal or adequate to the need. As noted earlier, in the absence of meaningful access to community-based treatment, it is difficult to ensure access to opioid pharmacotherapy upon release.

Even if adequate funding for pharmacotherapy was available, there are additional regulatory barriers associated with providing opioid agonist treatment in the community in the US that must also be overcome in correctional settings. For example, methadone treatment requires an OTP license and the delivery of bundled services of directly observed medication and counseling. Buprenorphine treatment requires physicians to obtain specialized training and a special federal approval to permit prescribing this medication.

Likewise, even when there are adequate treatment facilities in the community, corrections' officials may pose roadblocks to the implementation of pharmacological treatment for addiction. Reasons provided for not implementing pharmacotherapy include stigma, lack of understanding of the use of medications, philosophical objections to the use of medications, fear of diversion, and cost. ${ }^{4,7,19}$ Because state corrections' budgets are distinct from the budgets of the health care system, the potential benefits of starting medication during incarceration may accrue in the community to the benefit of the health system or the police, but would be borne by the correctional system. Thus, the implementation of pharmacotherapy for opioid dependence treatment in a correctional institution may be considered a financial burden to institution administrators.

Although surmountable, there are also barriers to conducting research on treatment for inmates. In the US, federal research regulations require that the local institutional review board apply to the Federal Office of Human Research
Protection to ensure that special conditions are met prior to initiating research with prisoners, including permitting only certain types of research and requiring membership of a prisoner advocate on the local institutional review board. Despite their status as prisoners, participation in research, of course, must be voluntary and free of coercion. Payment for participation in health research in prison is possible ${ }^{42}$ but must be done with care to avoid the payment seeming coercive in a resource-poor environment. Many of the papers reviewed herein did not describe how these issues were dealt with, and future research papers should provide a description of how the study addressed them.

The RCTs reviewed have a number of limitations that should be addressed in future research. Three of the eight RCTs had $<100$ participants. . $^{18,32,36}$ Future studies would be strengthened by an adequate sample size, by clearly defined primary and secondary outcomes, and by addressing statistical power. Three of the trials did not conduct biological drug testing. ${ }^{18,23,26}$ Drug testing at follow-up provides objective data, which would strengthen reliability of self-reported drug use. Three of the studies reported postrelease outcomes only in the short term (ie, $<3$ months). ${ }^{23,33,36}$ Future research should include longer term follow-up of a year or more post release. Another limitation of the extant research is its focus on males, with five of the studies including only male participants. ${ }^{15,18,24,26,36}$ The cost to society from crime and incarceration and health-related costs associated with drug use underscore the need for economic analyses to accompany research whenever possible. This will help to bolster and make a compelling case to policy makers who might not be swayed solely on the basis of patient outcomes data.

Only one RCT examined the effectiveness of continuing opioid agonist treatment (in this case, methadone) compared to discontinuing it during incarceration. ${ }^{23}$ Continuing medications during detention while an individual is awaiting trial should be a minimum standard of care for any chronic illness (such as epilepsy or hypertension). However, this minimum standard is routinely violated in the US for opioid agonist treatment. There would not appear to be the need for additional research to demonstrate the benefits of continuing opioid agonist medication during detention given the robust evidence of its effectiveness in the community. However, future implementation studies examining approaches to maximizing return to care post release, including engagement strategies for those who do not reenter treatment, are needed.

Given the large number of opioid-dependent individuals who are out of treatment at the time of arrest and cycle in and out of jails, it is surprising that only one $\mathrm{RCT}^{26}$ has examined 
the use of opioid agonist treatment for out-of-treatment individuals at the time of incarceration. However, this study compared methadone to buprenorphine and lacked a treatment-as-usual group, making it difficult to draw comparisons to usual care. To address the lack of a comparison condition resembling (though superior to) usual care, our group is currently conducting a three-group random assignment study comparing initiating methadone in jail, initiating methadone in jail with postrelease patient navigation, and initiating methadone dose taper in jail with drug treatment and overdose prevention referral among newly arrested detainees in opioid withdrawal (NCT 02334215). This study, part of a cooperative study with two other sites that are examining XR-NTX prior to release, seeks to enroll 300 male and female participants and has a 1-month, 3-month, 6-month, and 12-month followup, and includes urine drug testing and economic analyses to address some of the limitations described earlier.

Finally, the opioid antagonist XR-NTX may prove quite useful for select individuals who prefer a long-acting, nonagonist medication for relapse prevention. However, given that the Lee et $\mathrm{al}^{36}$ study was a pilot, more research is needed in this area. There are currently two trials underway as part of the aforementioned cooperative study in Albuquerque, NM and New York, NY (NCT 02110264 and 01999946 , respectively) to compare XR-NTX to referral to treatment and overdose prevention that should provide additional information regarding the boundaries of this pharmacotherapy. Here, too, the research, even if strongly supportive of this approach, will have limited impact unless there is the possibility to continued treatment in the communities to which these individuals return.

\section{Acknowledgments}

This work was supported by the National Institute on Drug Abuse grant 5U01DA013636 (Schwartz PI). National Institute on Drug Abuse had no role in the collection, analysis, and interpretation of the data or in the writing of this manuscript or the decision to submit for publication. The authors wish to thank Dr Jerome H Jaffe for his critical review of the manuscript.

\section{Disclosure}

Ms Sharma and Drs Mitchell, Kelly, and Gryczynski report no conflicts of interest in this work. Dr Schwartz has in the past provided a one-time consultation to Reckitt Benckiser Pharmaceuticals on behalf of his employer Friends Research Institute. Dr O'Grady has in the past received reimbursement for his time from Reckitt Benckiser Pharmaceuticals.
Neither Reckitt Benckiser nor its now-separate pharmaceutical division, Indivior, had any role in the collection or interpretation of the data, in the writing of the manuscript, or in the decision to submit for publication. The authors report no other conflicts of interest in this work.

\section{References}

1. World Health Organization. Guidelines for the Psychosocially Assisted Pharmacological Treatment of Opioid Dependence. Geneva: World Health Organization: Department of Mental Health, Substance Abuse, World Health Organization, International Narcotics Control Board, United Nations Office on Drugs, and Crime; 2009.

2. Rich JD, Boutwell AE, Shield DC, et al. Attitudes and practices regarding the use of methadone in US state and federal prisons. $J$ Urban Health. 2005;82(3):411-419.

3. Substance Abuse and Mental Health Services Administration. Mental Health Services Administration Results from the 2012 National Survey on Drug Use and Health: Summary of National Findings. Rockville, MD: Substance Abuse and Mental Health Services Administration; 2013. [NSDUH Series H-46, HHS Publication No. (SMA) 13-4795; 2013].

4. Chandler RK, Fletcher BW, Volkow ND. Treating drug abuse and addiction in the criminal justice system: improving public health and safety. JAMA. 2009;301(2):183-190.

5. Mumola CJ, Karberg JC. Drug Use and Dependence, State and Federal Prisoners, 2004. Washington, DC: Department of Justice, Bureau of Justice Statistics (NCJ 213530); 2006.

6. Nunn A, Zaller N, Dickman S, Trimbur C, Nijhawan A, Rich JD. Methadone and buprenorphine prescribing and referral practices in US prison systems: results from a nationwide survey. Drug Alcohol Depend. 2009;105(1-2):83-88.

7. Dolan K, Khoei EM, Brentari C, Stevens A. Prisons and Drugs: A Global Review of Incarceration, Drug Use and Drug Services. Oxford: Beckley Foundation; 2007.

8. Sibbald B. Methadone maintenance expands inside federal prisons. CMAJ. 2002;167(10):1154.

9. Commission of the European Communities (CEC). Report from the Commission to the European Parliament and the Council on the Implementation of the Council Recommendation of 18 June 2003 on the Prevention and Reduction of Health-Related Harm Associated with Drug Dependence. Brussels: Commission of the European Communities; 2007.

10. Møller L, Gatherer A, Jürgens R, Stöver H, Nikogosian H. Health in Prisons: A WHO Guide to the Essentials in Prison Health. Copenhagen: WHO Regional Office Europe; 2007.

11. Stallwitz A, Stover H. The impact of substitution treatment in prisons - a literature review. Int J Drug Policy. 2007;18(6):464-474.

12. Zamani S, Farnia M, Torknejad A, et al. Patterns of drug use and HIVrelated risk behaviors among incarcerated people in a prison in Iran. J Urban Health. 2010;87(4):603-616.

13. Hicks J [webpage on the Internet]. Jailed Heroin Addicts in Maryland Can Get Treatment under New Program. The Washington Post; 2015. Available from: http://www.highbeam.com/doc/1P2-38369234.html? Accessed July 15, 2015

14. Mark TL, Woody GE, Juday T, Kleber HD. The economic costs of heroin addiction in the United States. Drug Alcohol Depend. 2001;61(2):195-206.

15. Kinlock TW, Gordon MS, Schwartz RP, Fitzgerald TT, O'Grady KE. A randomized clinical trial of methadone maintenance for prisoners: results at 12 months post-release. J Subst Abuse Treat. 2009;37(3):277-285.

16. Genberg BL, Astemborski J, Vlahov D, Kirk GD, Mehta SH. Incarceration and injection drug use in Baltimore, Maryland. Addiction. 2015;110(7):1152-1159. 
17. Binswanger IA, Blatchford PJ, Mueller SR, Stern MF. Mortality after prison release: opioid overdose and other causes of death, risk factors, and time trends from 1999 to 2009. Ann Intern Med. 2013;159(9): 592-600.

18. Dole VP, Robinson JW, Orraca J, Towns E, Searcy P, Caine E. Methadone treatment of randomly selected criminal addicts. $N$ Engl J Med. 1969;280(25):1372-1375.

19. Stöver H, Michels II. Drug use and opioid substitution treatment for prisoners. Harm Reduct J. 2010;7:17.

20. Hedrich D, Alves P, Farrell M, Stöver H, Møller L, Mayet S. The effectiveness of opioid maintenance treatment in prison settings: a systematic review. Addiction. 2012;107(3):501-517.

21. Lobmaier PP, Kunøe N, Gossop M, Katevoll T, Waal H. Naltrexone implants compared to methadone: outcomes six months after prison release. Eur Addict Res. 2010;16(3):139-145.

22. Kinlock TW, Battjes RJ, Schwartz RP, MTC Project Team. A novel opioid maintenance program for prisoners: report of post-release outcomes. Am J Drug Alcohol Abuse. 2005;31(3):433-454.

23. Rich JD, McKenzie M, Larney S, et al. Methadone continuation versus forced withdrawal on incarceration in a combined US prison and jail: a randomised, open-label trial. Lancet. 2015;386(9991):350-359.

24. Dolan KA, Shearer J, MacDonald M, Mattick RP, Hall W, Wodak AD. A randomised controlled trial of methadone maintenance treatment versus wait list control in an Australian prison system. Drug Alcohol Depend. 2003;72(1):59-65.

25. Dolan KA, Shearer J, White B, Zhou J, Kaldor J, Wodak AD. Fouryear follow-up of imprisoned male heroin users and methadone treatment: mortality, re-incarceration and hepatitis $\mathrm{C}$ infection. Addiction. 2005;100(6):820-828.

26. Magura S, Lee JD, Hershberger J, et al. Buprenorphine and methadone maintenance in jail and post-release: a randomized clinical trial. Drug Alcohol Depend. 2009;99(1-3):222-230.

27. Tomasino V, Swanson AJ, Nolan J, Shuman M. The key extended entry program (KEEP): a methadone treatment program for opiate-dependent inmates. Mt Sinai J Med. 2001;68(1):14-20.

28. Magura S, Rosenblum A, Lewis C, Joseph H. The effectiveness of in-jail methadone maintenance. J Drug Issues. 1993;23:75-99.

29. Marzo JN, Rotily M, Meroueh F, et al. Maintenance therapy and 3-year outcome of opioid-dependent prisoners: a prospective study in France (2003-06). Addiction. 2009;104(7):1233-1240.

30. Favrod-Coune T, Baroudi M, Casillas A, et al. Opioid substitution treatment in pretrial prison detention: a case study from Geneva, Switzerland. Swiss Med Wkly. 2013;143:w13898.
31. Kinlock TW, Gordon MS, Schwartz RP, O'Grady K, Fitzgerald TT, Wilson M. A randomized clinical trial of methadone maintenance for prisoners: results at 1-month post-release. Drug Alcohol Depend. 2007;91(2-3):220-227.

32. McKenzie M, Zaller N, Dickman SL, et al. A randomized trial of methadone initiation prior to release from incarceration. Subst Abus. 2012;33(1):19-29.

33. Gordon MS, Kinlock TW, Schwartz RP, Fitzgerald TT, O'Grady KE, Vocci FJ. A randomized controlled trial of prison-initiated buprenorphine: prison outcomes and community treatment entry. Drug Alcohol Depend. 2014;142:33-40.

34. Garcia CA, Correa GC, Hernandez Viver AD, et al. Buprenorphinenaloxone treatment for pre-release opioid-dependent inmate in Puerto Rico. J Addict Med. 2007;1(3):126-132.

35. Zaller N, McKenzie M, Friedmann PD, Green TC, McGowan S, Rich JD. Initiation of buprenorphine during incarceration and retention in treatment upon release. J Subst Abuse Treat. 2013;45(2):222-226.

36. Lee JD, McDonald R, Grossman E, et al. Opioid treatment at release from jail using extended-release naltrexone: a pilot proof-of-concept randomized effectiveness trial. Addiction. 2015;110(6):1008-1014.

37. Gordon MS, Kinlock TW, Vocci FJ, Fitzgerald TT, Memisoglu A, Silverman B. A phase 4, pilot, open-label study of VIVITROL (Extended-Release Naltrexone XR-NTX) for prisoners. J Subst Abuse Treat. 2015;59:52-58.

38. GOV.UK [webpage on the Internet]. Prison Life. 2015. Available from: https://www.gov.uk/life-in-prison/healthcare-in-prison. Accessed November 9, 2015.

39. Wilper AP, Woolhandler S, Boyd JW, et al. The health and health care of US prisoners: results of a nationwide survey. Am J Public Health. 2009;99(4):666-672.

40. Boucher R. The case for methadone maintenance treatment in prisons. Vt L Rev. 2003;27:453-482.

41. Legal Action Center [webpage on the Internet]. Legality of Denying Access to Medication Assisted Treatment in the Criminal Justice System. 2011. Available from: http://lac.org/wp-content/uploads/2014/12/ MAT_Report_FINAL_12-1-2011.pdf. Accessed October 14, 2015.

42. Smoyer AB, Blankenship KM, Belt B. Compensation for incarcerated research participants: diverse state policies suggest a new research agenda. Am J Public Health. 2009;99(10):1746-1752.
Substance Abuse and Rehabilitation

\section{Publish your work in this journal}

Substance Abuse and Rehabilitation is an international, peer-reviewed, open access journal publishing original research, case reports, editorials, reviews and commentaries on all areas of addiction and substance abuse and options for treatment and rehabilitation. The manuscript management system is completely online and includes a very quick and fair

\section{Dovepress}

peer-review system. Visit http://www.dovepress.com/testimonials.php to read real quotes from published authors. 